\title{
Relationship Between Plasma Total Homocysteine Levels and Mean Corrected TIMI Frame Count in Patients with Acute Myocardial Infarction
}

\author{
Feng $\mathrm{Hu}$ (D) \\ Feng $\mathrm{Lu}^{2}$ \\ Xiao Huang (D) \\ Xiaoshu Cheng'
}

'The Department of Cardiovascular Medicine, The Second Affiliated Hospital of Nanchang University, Nanchang, People's Republic of China; ${ }^{2}$ The Department of Cardiothoracic Surgery, The Second Affiliated Hospital of Nanchang University, Nanchang, People's Republic of China
Correspondence: Xiao Huang;

Xiaoshu Cheng

Department of Cardiovascular Medicine, The Second Affiliated Hospital of

Nanchang University, No. I Minde Road, Nanchang, 330006, Jiangxi, People's

Republic of China

$\mathrm{Tel}+86 \mid 3607089128$

Fax +86-791-86262262

Email drxiaohuang@163.com;

xiaoshumenfanI26@I63.com
Objective: To explore the relationship between the plasma total homocysteine (tHcy) levels and slow coronary flow (SCF) measured by mean corrected TIMI frame count (CTFC) in patients with acute myocardial infarction (AMI).

Methods: Ninety-one patients with primary myocardial infarction were enrolled in this study. The quantitative measurement of coronary blood flow was performed using the mean CTFC method. Plasma tHcy levels were determined using enzymatic assay from venous blood samples. Multivariable linear regression models indicated were used to estimate the effect size $(\beta)$ of plasma tHcy levels on mean CTFC levels.

Results: Compared with patients with the mean CTFC $\leq 23.61$ frames per second (FPS) group, there were increased plasma tHcy levels in patients of the second, third and highest mean CTFC quartiles $(P<0.001)$. Linear regression models indicated that plasma tHcy levels were positively associated with mean CTFC levels (adjusted- $\beta$ per $S D$ increase: 1.96 , $95 \%$ CI 1.20 to $2.73, P<0.001)$. Compared to the tHcy $\leq 12.30 \mu \mathrm{mol} / \mathrm{L}$ group, the third and highest tHcy-quartile groups had higher mean CTFC levels (adjusted- $\beta: 2.52,95 \%$ CI 0.39 to $4.65, P=0.023$; adjusted- $\beta$ : 5.07, 95\% CI 2.98 to 7.16, $P<0.001$, respectively; $P$ for trend $<0.001)$. Moreover, this positive relationship was modified by diabetes mellitus ( $P$-value for interaction was 0.046).

Conclusion: We found a positive relationship between plasma tHcy levels and mean CTFC levels in patients with AMI. Moreover, diabetes mellitus played an interactive role in this positive association between the plasma tHcy levels and mean CTFC levels.

Keywords: hyperhomocysteinemia, mean corrected TIMI frame count, acute myocardial infarction, slow coronary flow, coronary microcirculation dysfunction

\section{Introduction}

Slow coronary flow (SCF), a syndrome presenting with chest pain or tightness related to a delay in the progression of contrast injected into the distal coronary vasculature during coronary angiography, was first proposed in $1972 .{ }^{1}$ Although its pathogenesis is still not fully elucidated, it has been reported that coronary microcirculation dysfunction (CMD) was responsible for the development of SCF phenomenon. $^{1-3}$

In the setting of acute myocardial infarction (AMI) after primary percutaneous transluminal coronary intervention (PCI), CMD is an indicator of poor long-term clinical prognosis. ${ }^{4}$ There are multiple founded techniques to assess microvascular injury or dysfunction in the catheterization room. ${ }^{5}$ Corrected TIMI frame count 
(CTFC) is an angiographic argument that can re-stratify the risk of patients with post-procedural thrombolysis in myocardial infarction (TIMI) grade III flow and reflect the existence of SCF and CMD. ${ }^{6,7}$

Homocysteine is a sulfur-containing amino acid that functions as a key intermediate in methionine metabolism and hyperhomocysteinemia (HHcy) is well established risk factor for cardiovascular disease. $^{8}$ Angiographic findings ${ }^{9-16}$ found that patients with SCF had significantly higher plasma total homocysteine (tHcy) levels than those with normal coronary flow and HHcy was strongly correlated with SCF phenomenon in patients without organic coronary stenosis. In addition, HHcy was a potential predictor for SCF prognosis and related to cumulative cardiac events. ${ }^{14}$ Moreover, Li et $\mathrm{al}^{17}$ observed that increased plasma tHcy levels $(\geq 17.55 \mu \mathrm{mol} / \mathrm{L})$ were independent negative predictor of spontaneous reperfusion (defined as achievement of TIMI grade III flow in the infarct-related artery (IRA) before primary PCI) in patients with AMI, implying the potential relationship between HHcy with less early IRA patency. However, to our knowledge, there is no detailed study evaluating the relationship between plasma tHcy levels and SCF measured by mean CTFC in patients with AMI. Therefore, the aim of this study was to investigate the relationship between plasma tHcy levels and mean CTFC in patients with AMI.

\section{Methods}

\section{Study Population}

This single-center study enrolled 91 angiographically identified patients with AMI, admitted to chest pain center at the Second Affiliated Hospital of Nanchang University between July 2019 and December 2020. There were 60 patients with ST-segment elevation myocardial infarction (STEMI), 31 cases with non-ST-segment elevation myocardial infarction (NSTEMI) in our analysis. Patients with diagnosed coronary artery disease, history of myocardial infarction, left ventricular dysfunction (left ventricular ejection fraction $<50 \%$ ), severe heart valve disease, cardiomyopathy, arrhythmia, left ventricular hypertrophy, uncontrolled hypertension, diabetes mellitus, connective tissue disease and liver, kidney, thyroid dysfunction or vitamin intake were excluded from the study. Left ventricular ejection fraction (LVEF) and left ventricle end diastolic diameter (LVEDD) were collected and analyzed independently by a third part. Written approved informed consent was obtained from each participant. All procedures performed in studies involving human participants were in accordance with the ethical standards of the institutional and national research committee and with the 1964 Helsinki declaration and its later amendments or comparable ethical standards. The study was approved by the Ethical Review Committee of the Second Affiliated Hospital of Nanchang University (Number: 2018242).

\section{Coronary Angiography and Corrected TIMI Frame Count}

All enrolled patients were accepted urgent coronary angiography and we used iohexol as contrast agent. All angiograms were performed using Standard Judkin's left and right 6 French diagnostic catheters. Acquisition positions were right anterior oblique $30^{\circ}$ for the left anterior descending (LAD) and the left circumflex artery (LCX), and left anterior oblique $50^{\circ}$ for the right coronary artery (RCA). The speed of imaging collection was 15 frames per second (FPS). The angiographic analysis was performed retrospectively by an independent core lab using software of iMedical 8.0. Coronary flow rates of these subjects were measured by CTFC according to the means described by Gibson et al. ${ }^{6}$ In brief, the first frame was considered that where a column of dye extended across the entire width of the origin of the artery, touching both its borders with evidence of antegrade motion of the dye. The last frame was defined as the frame when the dye first entered a certain distal landmark branch of (but did not necessarily fill) each vessel. ${ }^{6}$ Because the LAD artery TFC is approximately 1.7- times greater than that of the LCX and RCA, the TIMI frame count (TFC) of the LAD was corrected by dividing by $1.7 .^{6}$ We calculated mean CTFC $=(\mathrm{LAD} \mathrm{TFC} / 1.7+\mathrm{LCX} \mathrm{TFC}+\mathrm{RCA} \mathrm{TFC}) / 3$. The subjects with mean CTFC greater than 27 FPS were accepted as having SCF in the present study according to previous reports. ${ }^{18}$ Furthermore, the risk of nosocomial death was assessed by Grace Score which stratified the risk of AMI patients and accurately predicted the mortality during hospitalization or in the long term. ${ }^{19}$ The severity of coronary artery lesions was evaluated by the Gensini scoring system according to the narrowing degree and functional significance of each coronary vessel. ${ }^{20}$

\section{Laboratory Assay}

Blood samples were collected utilizing venipuncture after an overnight fast of at least 12 hours. The levels of plasma tHcy were measured by enzymatic cycling assay analysis 
using a commercially available "Immulite Homocysteine" kit (Product ID: YZB/JI 0163-2013, Hebei aioulu biotechnology company, CHINA). The levels of B-type natriuretic peptide (BNP), hyper-sensitivity cTnI, blood urea nitrogen (BUN), estimated glomerular filtration rate (eGFR); serum creatinine and uric acid, white blood cells, hemoglobin, platelets, plasma albumin, total cholesterol (TC), total triglyceride (TG), low density lipoproteincholesterol (LDL-C), high-density lipoprotein-cholesterol (HDL), and non-high-density lipoprotein cholesterol (NHDLC) were measured using automatic clinical analyzers (Beckman Coulter). The estimated glomerular filtration rate (eGFR) was calculated by the equation of Chronic Kidney Disease Epidemiology Collaboration (CKD-EPI).

\section{Statistical Analysis}

Continuous variables are presented as the means \pm standard deviation $(S D)$ or the median (quartiles), as appropriate, and are compared using the Student's $t$-test, one-way analysis or the Mann Whitney $U$-test, depending on whether the quantitative data were consistent with a normal distribution. Categorical variables were presented as count (percentage), differences between groups were measured by chi-square test.

Linear regression models indicated were used to estimate the effect size $(\beta)$ of plasma tHcy levels on mean CTFC levels. The crude model was not adjusted for any confounder. The model I was only adjusted for age and sex. The model II was confounder model. The confounder model screened covariates including age, sex, systolic blood pressure (SBP), diastolic blood pressure (DBP), heart rate (HR), current smoking and drinking status, the type of AMI, Killip classification, hypertension, diabetes mellitus, TC, TG, HDL-C, LDL-C, NHDLC, serum uric acid, eGFR, LVEF, Grace risk scores and Gensini coronary lesion scores. We selected these confounders on the basis of their associations with the outcomes of interest more than $10 \%$. We considered the confounder model to be the main model.

Furthermore, the generalized additive model and smooth curve fitting (penalized spline method) was used to visually show the relationship between plasma tHcy levels and mean CTFC levels.

All statistical analyses were performed using the statistical package $\mathrm{R}$ (http://www.R-project.org, The $R$ Foundation, version 3.4.3) and the Empower (R; www. empowerstats.com; X\&Y Solutions, Inc, Boston, MA,
USA). All P-values are two-tailed, and $\mathrm{P}<0.05$ was considered to be statistically significant.

\section{Results \\ Patient Characteristics}

The present study included 91 AMI patients (age: $65.34 \pm$ 11.12 years, range $38-86$ years; male, $75.82 \%$ ). The clinical characteristics of the study participants were presented grouped by quartiles of mean CTFC. There were no significant differences between the groups in terms of clinical parameters other than the plasma tHcy levels (Table 1). Compared with patients with mean CTFC $\leq 23.61$ FPS, there were increased plasma tHcy levels in patients of the second, third and highest mean CTFC quartiles $(P<$ 0.001 , Table 1). Furthermore, compared with patients with mean CTFC $\leq 23.61$ FPS, there were increased numbers of slow flow arteries in patients of the third and highest mean CTFC quartiles $(P<0.001$, Table 2$)$. However, there were no significant differences between the groups in terms of Gensini score of epicardial coronary artery lesion and Grace score of nosocomial death risk $(P>0.05$, Table 2$)$.

\section{Association Between Plasma tHcy Levels and Mean CTFC Levels}

Linear regression models indicated that plasma tHcy levels were positively associated with mean CTFC levels (adjusted- $\beta$ per $S D$ increase: $1.96,95 \%$ CI 1.20 to 2.73 , $P<0.001$; Table 3). Compared without HHcy group (Hcy $<15 \mu \mathrm{mol} / \mathrm{L}$ ), patients with HHcy had higher mean CTFC levels (adjusted- $\beta$ : $2.89,95 \%$ CI 1.35 to $4.43, P<0.001$; Table 3). Compared to the $\mathrm{tHcy} \leq 12.30 \mu \mathrm{mol} / \mathrm{L}$ group, the third and highest tHcy-quartile groups had higher mean CTFC levels (adjusted- $\beta$ : $2.52,95 \%$ CI 0.39 to $4.65, P=$ 0.023 ; adjusted- $\beta$ : $5.07,95 \%$ CI 2.98 to $7.16, P<0.001$, respectively; $P$ for trend $<0.001$; Table 3 ). The smooth curve fitting further confirmed this positive relationship between plasma tHcy levels and mean CTFC levels (Figure 1).

\section{Subgroup Analyses by Potential Effect Modifiers}

To explore whether this positive correlation between plasma tHcy levels and mean CTFC levels was still stable in different subgroups, we conducted stratified and interaction analyses. We found that this association between plasma tHcy levels and mean CTFC levels was modified by diabetes mellitus ( $P$-value for interaction was 0.046 , Figure 2$)$. There were not 


\begin{tabular}{|c|c|c|c|c|c|c|c|c|c|c|c|c|c|c|c|c|c|c|}
\hline 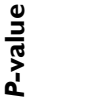 & जิ & హ్ & $\begin{array}{l}\text { No. } \\
0\end{array}$ & $\begin{array}{l}\text { مू } \\
\text { ơ } \\
0\end{array}$ & $\begin{array}{l}\text { do } \\
\text { o. } \\
\text { o. }\end{array}$ & $\frac{0}{0}$ & $\begin{array}{l}\infty \\
\stackrel{0}{0} \\
\stackrel{0}{0}\end{array}$ & $\frac{\stackrel{\circ}{\frac{m}{0}}}{.0}$ & $\frac{\hat{m}}{\mathrm{~m}}$ & $\frac{0}{0}$ & $\begin{array}{l}\stackrel{\omega}{0} \\
\hat{n} \\
0\end{array}$ & 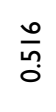 & $\begin{array}{l}\infty \\
\stackrel{\infty}{0} \\
0\end{array}$ & $\begin{array}{l}\bar{\delta} \\
\dot{Q} \\
\dot{v}\end{array}$ & స్ & 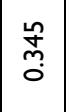 & $\frac{\tilde{m}}{0}$ & $\begin{array}{l}\hat{\circ} \\
\text { مै }\end{array}$ \\
\hline 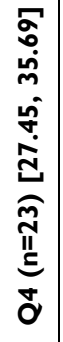 & 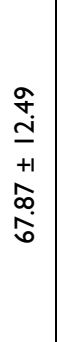 & 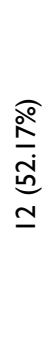 & 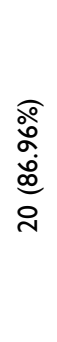 & 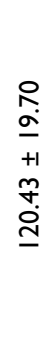 & 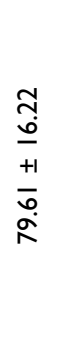 & 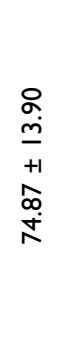 & $\begin{array}{l}\frac{\sigma}{\circ} \\
\stackrel{a}{\infty} \\
=\end{array}$ & 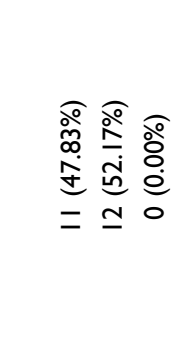 & 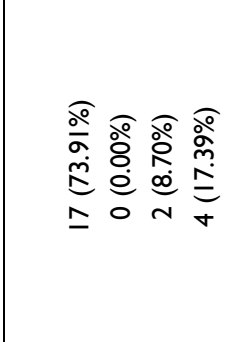 & 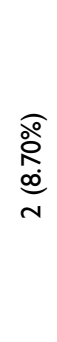 & 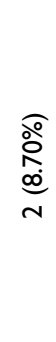 & $\begin{array}{l}\text { o } \\
\text { o } \\
\text { ¿े } \\
0 \\
0\end{array}$ & $\frac{\substack{\frac{o}{N} \\
\frac{1}{2}}}{n}$ & 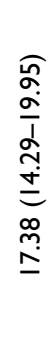 & 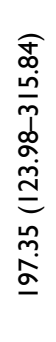 & 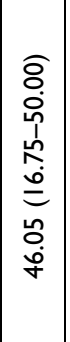 & 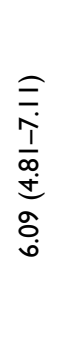 & 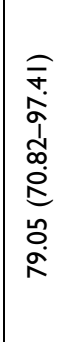 \\
\hline 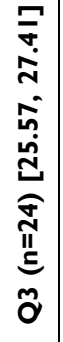 & 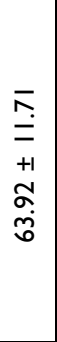 & 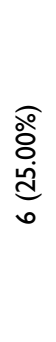 & 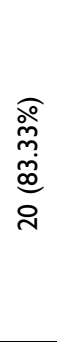 & 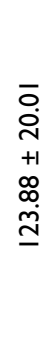 & 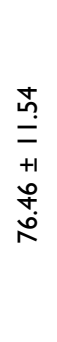 & 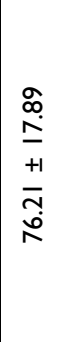 & 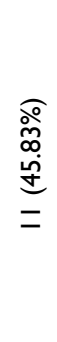 & 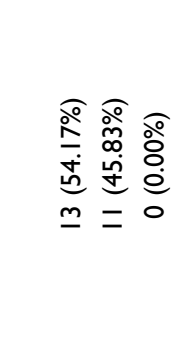 & 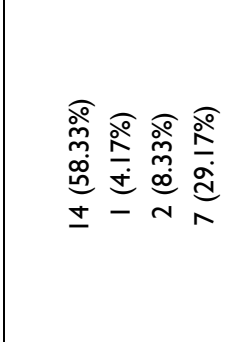 & 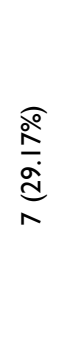 & $\begin{array}{c}\stackrel{\curvearrowright}{\circ} \\
\stackrel{m}{0} \\
\infty \\
\sim\end{array}$ & 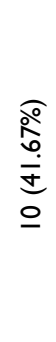 & 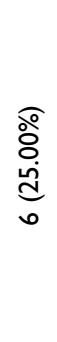 & 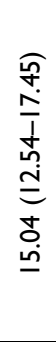 & 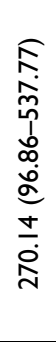 & 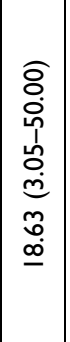 & 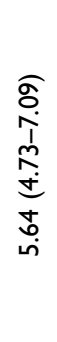 & 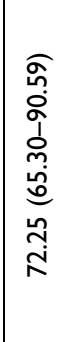 \\
\hline 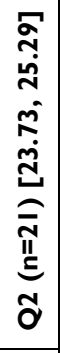 & $\begin{array}{l}\stackrel{0}{2} \\
\alpha \\
+1 \\
\infty \\
\infty \\
\tilde{n}\end{array}$ & 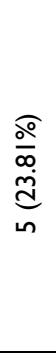 & $\begin{array}{l}\frac{\circ}{\circ} \\
\stackrel{0}{\circ} \\
0\end{array}$ & 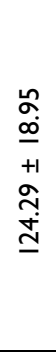 & 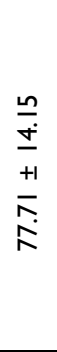 & 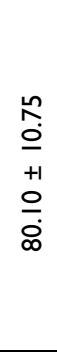 & 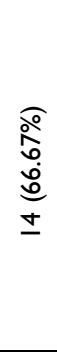 & 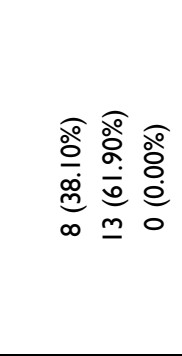 & 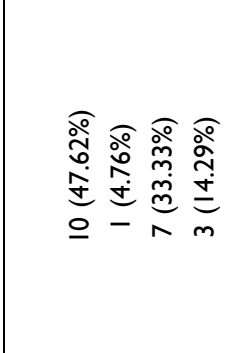 & 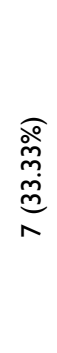 & 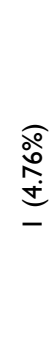 & 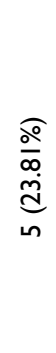 & 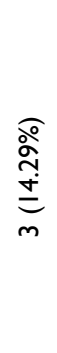 & 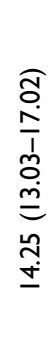 & 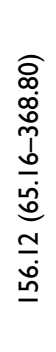 & 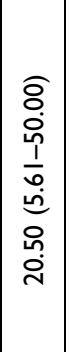 & 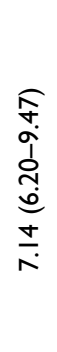 & 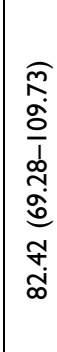 \\
\hline 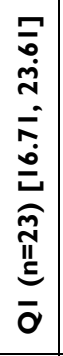 & $\begin{array}{l}0 \\
0 \\
0 \\
+1 \\
o \\
0 \\
0 \\
0\end{array}$ & $\begin{array}{l}\text { ò } \\
\text { ọ } \\
\text { 立 } \\
= \\
=\end{array}$ & 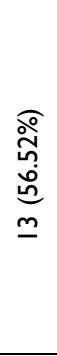 & 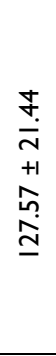 & 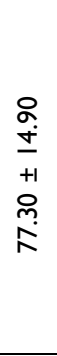 & 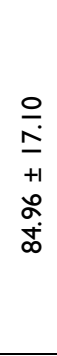 & 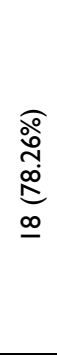 & 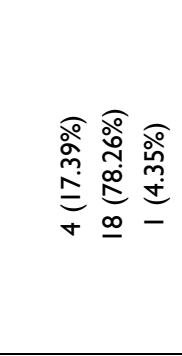 & 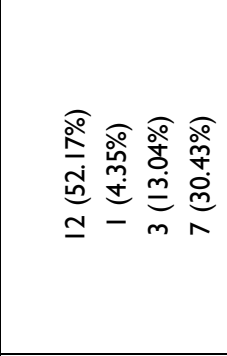 & $\begin{array}{l}\frac{\widehat{\rho}}{m} \\
\frac{0}{\infty} \\
a\end{array}$ & 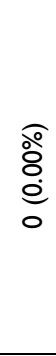 & 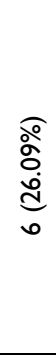 & 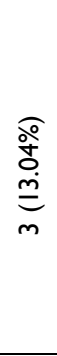 & 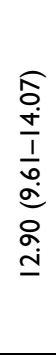 & 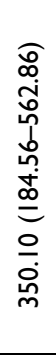 & 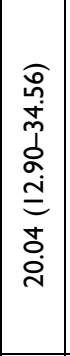 & 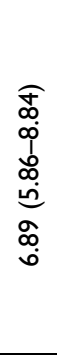 & 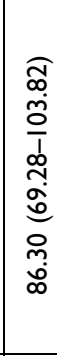 \\
\hline & 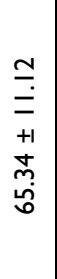 & 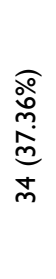 & 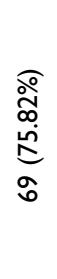 & 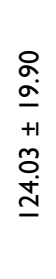 & $\begin{array}{l}0 \\
\dot{x} \\
+1 \\
+1 \\
0 \\
\stackrel{1}{N}\end{array}$ & 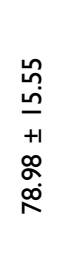 & 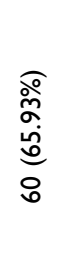 & 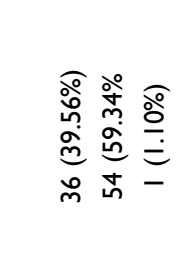 & 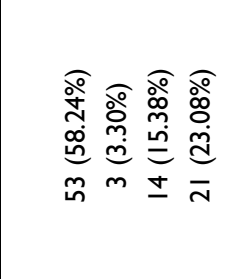 & 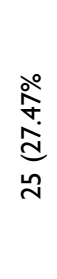 & 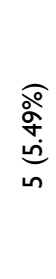 & 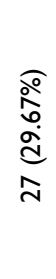 & $\begin{array}{l}\text { o } \\
\stackrel{0}{0} \\
\infty \\
\infty \\
\simeq\end{array}$ & 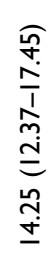 & 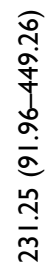 & 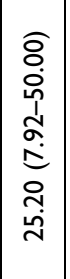 & 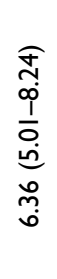 & 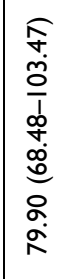 \\
\hline & 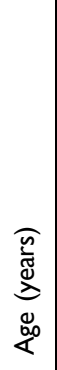 & 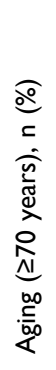 & 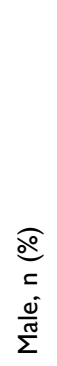 & 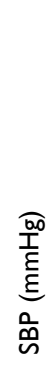 & 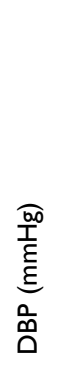 & 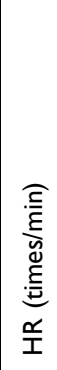 & 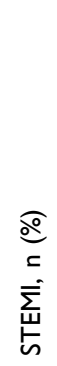 & 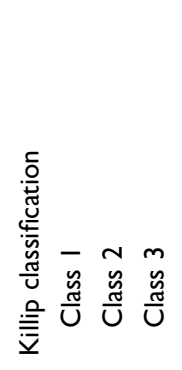 & 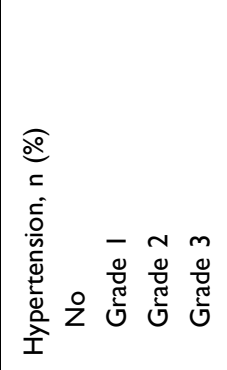 & 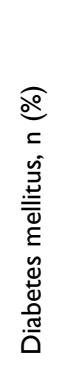 & $\begin{array}{l}\text { o } \\
\text { c } \\
0 \\
0 \\
0\end{array}$ & 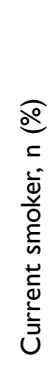 & 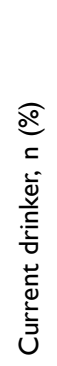 & 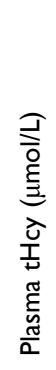 & 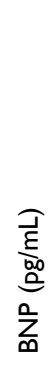 & 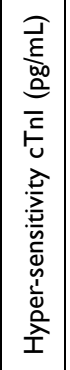 & 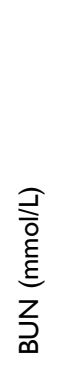 & 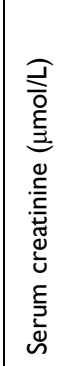 \\
\hline
\end{tabular}




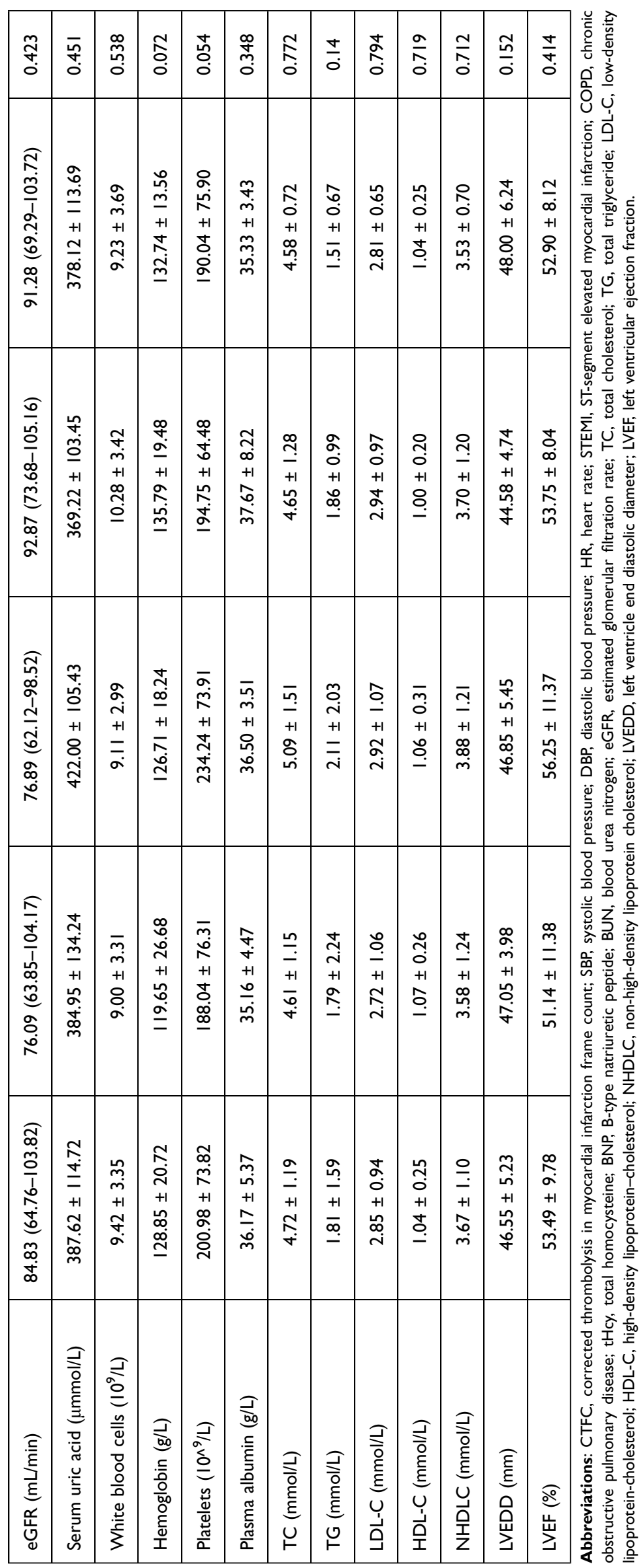




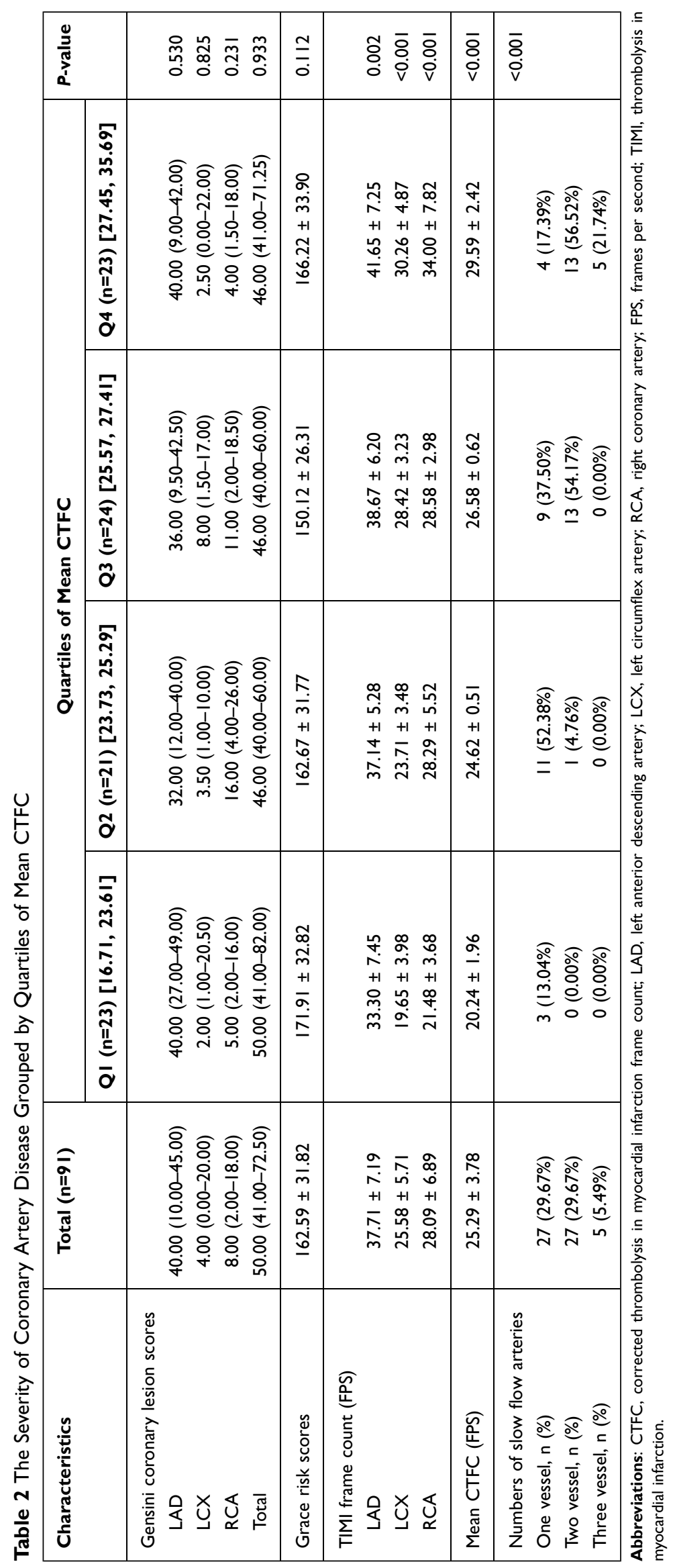




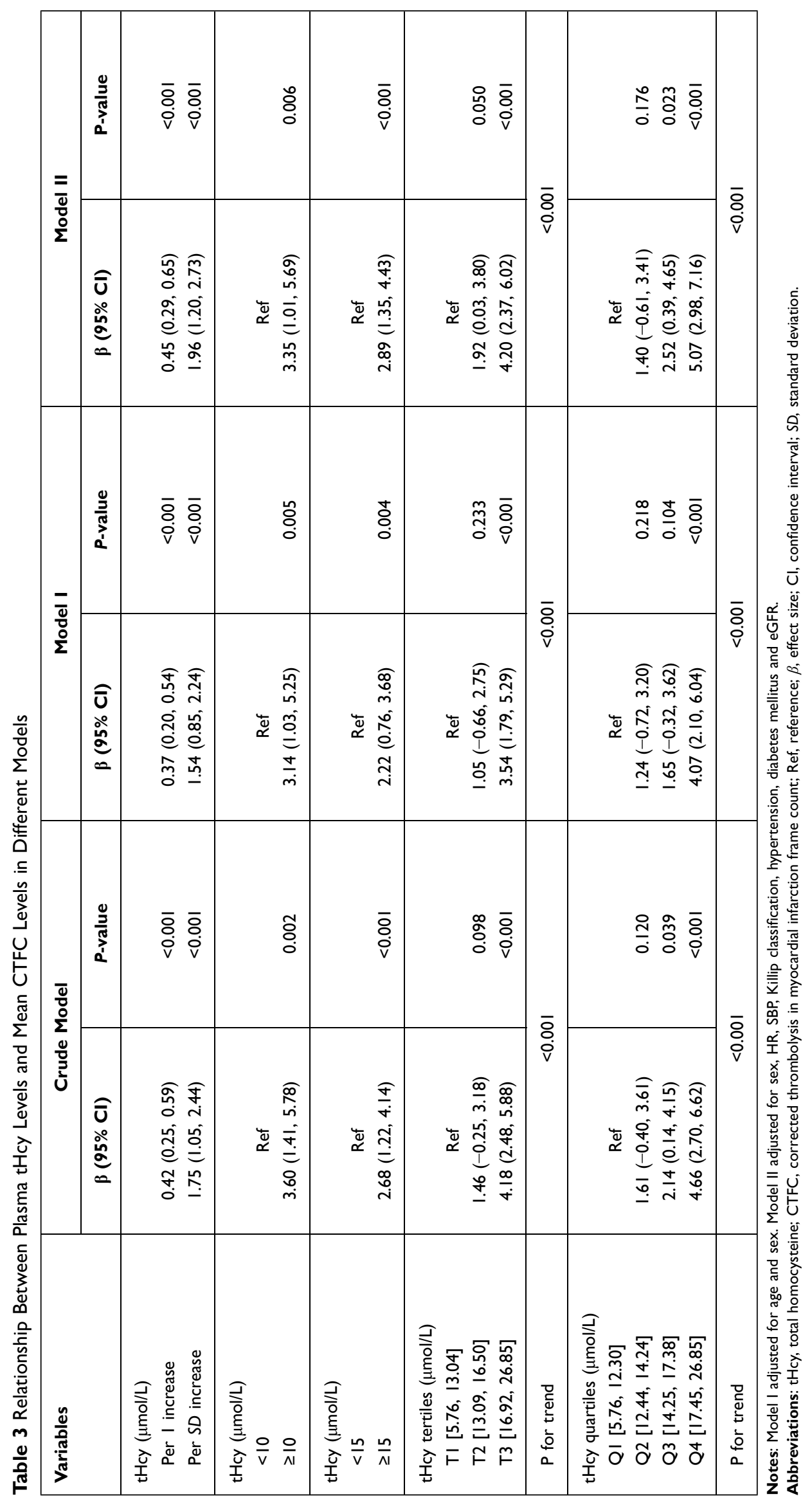




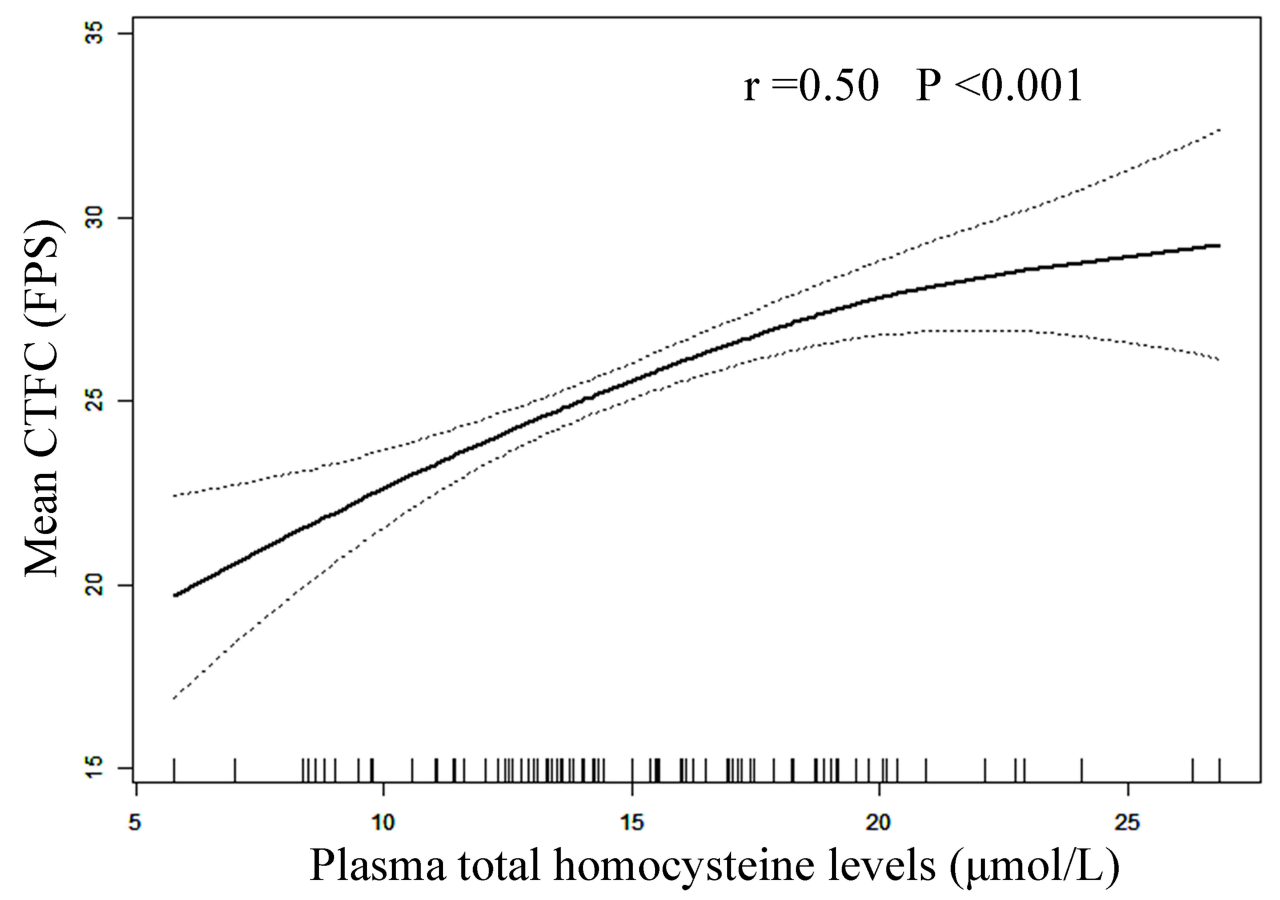

Figure I Smooth curve of correlation between plasma tHcy levels and mean CTFC levels. Smooth curve and $r$ adjusted for sex, HR, SBP, Killip classification, hypertension, diabetes mellitus and eGFR.

Abbreviations: CTFC, corrected thrombolysis in myocardial infarction frame count; FPS, frames per second; $r$, coefficient of partial correlation.

significant interactions in any of the following subgroups, including aging, sex (male vs female), SBP ( $<140$ vs $\geq 140$ mmHg), HR dichotomy ( $\leq 75$ vs $\geq 76$ times/min), AMI (NSTEMI vs STEMI), current smoker (no vs yes), eGFR dichotomy ( $\leq 82.3 \mathrm{vs} \geq 84.8 \mathrm{~mL} / \mathrm{min} / 1.73 \mathrm{~m}^{\wedge^{2}}$ ) and Grace risk scores dichotomy ( $\leq 164 \mathrm{vs} \geq 165$ ) (all $P$-values for interaction respectively were greater than 0.05 , Figure 2 ).

\section{Discussion}

The present study showed that plasma tHcy levels were significantly positively associated with mean CTFC levels, a metric of reflecting SCF and CMD, in patients with AMI. Moreover, diabetes mellitus played an interactive role in this positive association between the plasma tHcy levels and mean CTFC levels.

The risk factors and pathophysiology for the appearance of SCF are still unclear, and different ethnic populations have different risk factors on account of the different prevalence rates of comorbidities and genetic variation regarding predisposition to SCF. In a study performed by Beltrame et $\mathrm{al}^{21}$ in an Australian population, male sex and smoking were found to be independent risk factors for SCF. In two studies done in North Indian subjects, body mass index ${ }^{18}$ or hypertension, dyslipidemia, smoking and tobacco chewing were independently positively associated with SCF phenomenon. ${ }^{22}$ A study done on Iranian population identified smoking, total cholesterol, hematocrit, fasting blood glucose and red cell distribution to be independent risk factors associated with $\mathrm{SCF}^{23}$ Demirci et $\mathrm{al}^{16}$ showed that patients with SCF had significantly higher plasma tHcy levels than those with normal coronary flow. Li et $\mathrm{al}^{14}$ found HHcy and mean platelet volume to be independent risk factors for SCF in a Chinese Han population. The coronary microvasculature is the dominant gatekeeper for myocardial blood flow below the visible epicardial arteries. The CMD can lead to angina pectoris which is prolonged or poorly responsive to nitrates and may persist despite successful revascularization, affect ventricular function and remodeling in the long term. ${ }^{24}$ The evidence suggests that CMD in AMI patients is associated with adverse long-term survival. ${ }^{4}$

The development of CMD in AMI patients involved multiple pathophysiological processes, especially for endothelial dysfunction (ED). ${ }^{5}$ Homocysteine is a possibly cytotoxic amino acid synthesized during methionine metabolic cycle. Celermajer et $\mathrm{al}^{25}$ found that HHcy was associated with impaired endothelial function of systemic arteries and decreased nitric oxide bioavailability in hyperhomocystinuria children. Tanriverdi et $\mathrm{al}^{13}$ showed that HHcy impaired endothelial function via 


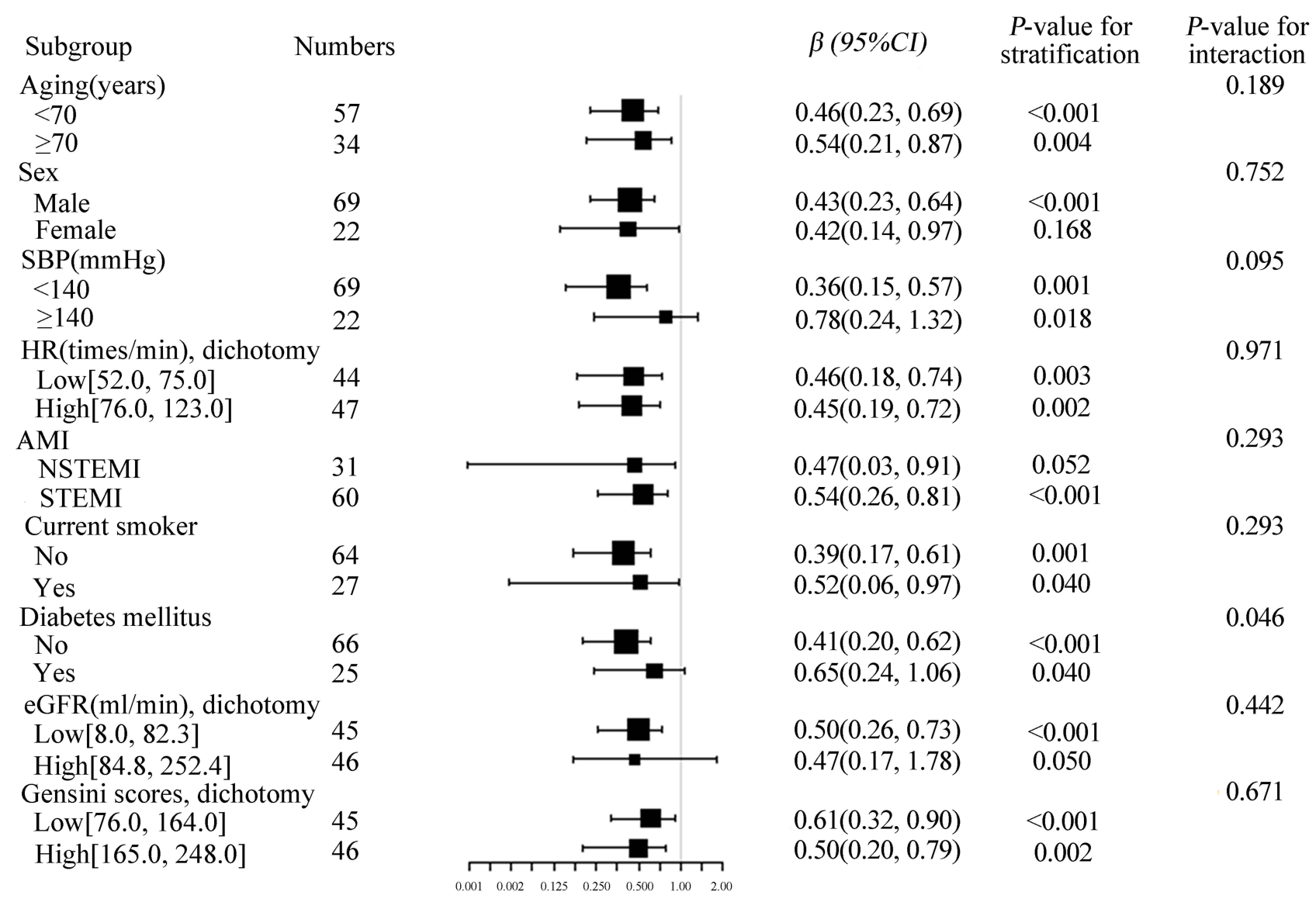

Figure 2 Effect size of plasma tHcy levels on mean CTFC in prespecified and exploratory subgroups. Each stratification adjusted for age, sex, HR, SBP, Killip classification, hypertension, diabetes mellitus and eGFR except the subgroup variable.

Abbreviations: SBP, systolic blood pressure; HR, heart rate; AMI, acute myocardial infarction; NSTEMI, non-ST-segment elevation myocardial infarction; STEMI, ST-segment elevation myocardial infarction; eGFR, estimated glomerular filtration rate; $\beta$, effect size; $\mathrm{Cl}$, confidence interval.

oxidative stress in SCF patients. Cheng et $\mathrm{al}^{26}$ found that HHcy impaired endothelium-dependent micro-vasculature relaxation to acetylcholine in mouse small mesenteric artery via oxidation of $\mathrm{SK}_{\mathrm{Ca}} / \mathrm{IK}_{\mathrm{Ca}}$. Intermediate $\mathrm{HHcy}$ potentiated diabetes impaired endothelium-derived hyperpolarizing factor-induced microvasculature relaxation via $\mathrm{H}_{2} \mathrm{~S}$-downregulation and $\mathrm{IK}_{\mathrm{Ca}}$ tyrosine nitration in T2DM/ HHcy mice. ${ }^{27}$ Otherwise, recent research showed HHcy could cause mitochondrial dysfunction and tissue damage mostly by inhibiting mitochondrial complex I gene expression which leads to impaired etransfer, complex assembly and redox homeostasis dysregulation in human disease and experimental models. ${ }^{28}$ Therefore, we speculated that HHcy might cause endothelial dysfunction or injury, which further result in CMD and SCF in AMI patients.

Moreover, previous studies have proved that plasma tHcy levels were relevant with coronary slow flow in patients without occlusive coronary arteries. $^{9-15}$ It's worth noting that partially differed from that of stable coronary artery disease, microvascular embolism or spasm might play a vital role due to severe thrombus burden on the onset of acute coronary events.

We found diabetes mellitus might play an interactive role in the association between the plasma tHcy levels and mean CTFC levels after adjusted all possible covariates. It is widely recognized that diabetes mellitus was associated with $\mathrm{ED}$ and $\mathrm{CMD}^{29}$ in animal models ${ }^{27,30,31}$ and clinical studies. ${ }^{32-36}$ Verma et al ${ }^{31}$ showed that diabetic microcirculatory disturbances were provoked by deposition of amyloid-forming amylin in red blood cells (RBC) and micro vasculatures, which thus altering RBC-capillary interaction leading to activation of hypoxia signaling pathways and pathologic erythropoiesis. Di et $\mathrm{al}^{32}$ found that markedly reduced and similar endothelium-dependent and -independent coronary vasodilator function in subjects with both type I and type II diabetes mellitus, by measuring myocardial blood flow measured in a different state using Positron emission tomography imaging. Von et $\mathrm{al}^{34}$ 
showed that type II diabetic patients had a reduced coronary flow reserve, especially with concomitant albuminuria, suggesting a common microvascular impairment occurring in multiple microvascular beds. But the $P$-value for interaction was 0.046 , belonging to marginal significance. Therefore, more researches with larger sample size is needed to prove this correlation.

In addition to the improvement of pharmacological and interventional therapy, the integrated management of CMD in AMI patients should incorporate lifestyle modification and the control of numerous risk factors, such as tHcy-lowering therapy, hypoglycaemic therapy, and cardiac rehabilitation therapy. Treatment combining drug therapy and cardiac rehabilitation could reduce the plasma levels of high-sensitivity C-reactive protein, homocysteine and arginine, significantly improve the symptoms of SCF phenomenon and increase coronary blood flow velocity. ${ }^{37}$

\section{Limitations}

Our study has some limitations. First, mean CTFC is not that stability because of the difference between physiological status, operator technique, the force of injection and catheter engagement. Second, the angiographic definition of SCF relies on axial contrast angiograms of the vessel lumen. Hence, future studies call for establish a diagnostic criteria for CMD using precise verified techniques, such as coronary flow velocity reserve (CFVR), cardiovascular magnetic resonance myocardial perfusion imaging and Index of microvascular resistance (IMR). ${ }^{7,38,39}$ Moreover, another restriction is that plasma levels of the folic acid, vitamin B12 and helicobacter pylori infection ${ }^{40}$ as well as methylenetetrahydrofolate reductase gene polymorphism were not able to be surveyed to determine the possible disturbance of methionine metabolism. ${ }^{15}$ Finally, in consideration of the small sample size in our study, further studies with larger samples are needed to confirm our results and to evaluate the effectiveness of homocysteine reducing treatment.

\section{Conclusions}

In conclusion, we found a positive relationship between plasma tHcy levels and mean CTFC levels in patients with AMI. HHcy may play an important role in the pathogenesis of SCF in patients with AMI. Moreover, diabetes mellitus played an interactive role in this positive association between the plasma tHcy levels and mean CTFC levels.

\section{Data Sharing Statement}

The data used to support the findings of this study are available from the corresponding author on request.

\section{Ethics Approval}

Written approved informed consent was obtained from each participant. All procedures performed in studies involving human participants were in accordance with the ethical standards of the institutional and national research committee and with the 1964 Helsinki declaration and its later amendments or comparable ethical standards. The study was approved by the Ethical Review Committee of the Second Affiliated Hospital of Nanchang University (Number: 2018242).

\section{Funding}

The study was supported by funding from the following: the National Natural Science Foundation of China [81960074].

\section{Disclosure}

The authors have no conflicts of interest regarding this work or the publication of this paper.

\section{References}

1. Tambe AA, Demany MA, Zimmerman HA, et al. Angina pectoris and slow flow velocity of dye in coronary arteries - a new angiographic finding. Am Heart J. 1972;84(1):66-71. doi:10.1016/0002-8703(72) 90307-9

2. Mangieri E, Macchiarelli G, Ciavolella M, et al. Slow coronary flow: clinical and histopathological features in patients with otherwise normal epicardial coronary arteries. Cathet Cardiovasc Diagn. 1996;37 (4):375-381. doi:10.1002/(SICI)1097-0304(199604)37:4<375::AIDCCD7>3.0.CO;2-8

3. Mosseri M, Yarom R, Gotsman MS, et al. Histologic evidence for small-vessel coronary artery disease in patients with angina pectoris and patent large coronary arteries. Circulation. 1986;74(5):964-972. doi:10.1161/01.CIR.74.5.964

4. Papapostolou S, Andrianopoulos N, Duffy SJ, et al. Long-term clinical outcomes of transient and persistent no-reflow following percutaneous coronary intervention (PCI): a multicentre Australian registry. EuroIntervention. 2018;14(2):185-193. doi:10.4244/EIJD-17-00269

5. Armstrong R, De Maria GL, Scarsini R, et al. Assessing and managing coronary microcirculation dysfunction in acute ST-segment elevation myocardial infarction. Expert Rev Cardiovasc Ther. 2019;17 (2):111-126. doi:10.1080/14779072.2019.1561279

6. Gibson CM, Cannon CP, Daley WL, et al. TIMI frame count: a quantitative method of assessing coronary artery flow. Circulation. 1996;93(5):879-888. doi:10.1161/01.CIR.93.5.879

7. Vijayan S, Barmby DS, Pearson IR, et al. Assessing coronary blood flow physiology in the cardiac catheterisation laboratory. Curr Cardiol Rev. 2017;13(3):232-243. doi:10.2174/1573403X1366617052510 2618 
8. Fu Y, Wang X, Kong W. Hyperhomocysteinaemia and vascular injury: advances in mechanisms and drug targets. $\mathrm{Br} J$ Pharmacol. 2018;175(8):1173-1189. doi:10.1111/bph.13988

9. Riza Erbay A, Turhan H, Yasar AS, et al. Elevated level of plasma homocysteine in patients with slow coronary flow. Int $J$ Cardiol. 2005;102(3):419-423. doi:10.1016/j.ijcard.2004.05.064

10. Barutcu I, Sezgin AT, Sezgin N, et al. Elevated plasma homocysteine level in slow coronary flow. Int $J$ Cardiol. 2005;101(1):143-145. doi:10.1016/j.ijcard.2004.01.030

11. Yurtdaş M, Özcan IT, Seyis AS, et al. Plasma homocysteine is associated with ischemic findings without organic stenosis in patients with slow coronary flow. $J$ Cardiol. 2013;61(2):138-143. doi:10.1016/j.jjcc.2012.10.001

12. Alihanoglu YI, Yildiz BS, Özcan EE, et al. Impaired coronary blood flow may be related to elevated homocysteine levels in patients with metabolic syndrome. Wien Klin Wochenschr. 2015;127(2122):864-870. doi:10.1007/s00508-015-0854-Z

13. Tanriverdi H, Evrengul H, Enli Y, et al. Effect of homocysteine-induced oxidative stress on endothelial function in coronary slow-flow. Cardiology. 2007;107(4):313-320. doi:10.1159/ 000099068

14. Li N, Tian L, Ren J, et al. Evaluation of homocysteine in the diagnosis and prognosis of coronary slow flow syndrome. Biomark Med. 2019;13(17):1439-1446. doi:10.2217/bmm-2018-0446

15. Tang O, Wu J, Qin F. Relationship between methylenetetrahydrofolate reductase gene polymorphism and the coronary slow flow phenomenon. Coron Artery Dis. 2014;25(8):653-657. doi:10.1097/ MCA.0000000000000151

16. Demirci E, Çelik O, Kalçık M, et al. Evaluation of homocystein and asymmetric dimethyl arginine levels in patients with coronary slow flow phenomenon. Interv Med Appl Sci. 2019;11:89-94.

17. Li J, Zhou Y, Zhang Y, et al. Admission homocysteine is an independent predictor of spontaneous reperfusion and early infarct-related artery patency before primary percutaneous coronary intervention in ST-segment elevation myocardial infarction. BMC Cardiovasc Disord. 2018;18(1):125. doi:10.1186/s12872-018-0868-3

18. Mukhopadhyay S, Kumar M, Yusuf J, et al. Risk factors and angiographic profile of coronary slow flow (CSF) phenomenon in North Indian population: an observational study. Indian Heart J. 2018;70 (3):405-409. doi:10.1016/j.ihj.2017.09.001

19. Fox KA, Anderson FA Jr, Dabbous OH, et al. Intervention in acute coronary syndromes: do patients undergo intervention on the basis of their risk characteristics? The Global Registry of Acute Coronary Events (GRACE). Heart. 2007;93(2):177-182. doi:10.1136/ hrt.2005.084830

20. Gensini GG. A more meaningful scoring system for determining the severity of coronary heart disease. Am J Cardiol. 1983;51(3):606. doi:10.1016/S0002-9149(83)80105-2

21. Beltrame JF, Limaye SB, Horowitz JD. The coronary slow flow phenomenon - a new coronary microvascular disorder. Cardiology. 2002;97(4):197-202. doi:10.1159/000063121

22. Sanghvi S, Mathur R, Baroopal A, et al. Clinical, demographic, risk factor and angiographic profile of coronary slow flow phenomenon: a single centre experience. Indian Heart J. 2018;70:S290-294. doi:10.1016/j.ihj.2018.06.001

23. Ghaffari S, Tajlil A, Aslanabadi N, et al. Clinical and laboratory predictors of coronary slow flow in coronary angiography. Perfusion. 2017;32(1):13-19. doi:10.1177/0267659116659918

24. Herrmann J, Kaski JC, Lerman A. Coronary microvascular dysfunction in the clinical setting: from mystery to reality. Eur Heart J. 2012;33(22):2771b-2782b. doi:10.1093/eurheartj/ehs246
25. Celermajer DS, Sorensen K, Ryalls M, et al. Impaired endothelial function occurs in the systemic arteries of children with homozygous homocystinuria but not in their heterozygous parents. $\mathrm{J} \mathrm{Am} \mathrm{Coll}$ Cardiol. 1993;22(3):854-858. doi:10.1016/0735-1097(93)90203-D

26. Cheng Z, Jiang X, Kruger WD, et al. Hyperhomocysteinemia impairs endothelium-derived hyperpolarizing factor-mediated vasorelaxation in transgenic cystathionine beta synthase-deficient mice. Blood. 2011;118:1998-2006.

27. Cheng Z, Shen X, Jiang X, et al. Hyperhomocysteinemia potentiates diabetes-impaired EDHF-induced vascular relaxation: role of insufficient hydrogen sulfide. Redox Biol. 2018;16:215-225. doi:10.1016/j. redox.2018.02.006

28. Cueto R, Zhang L, Shan HM, et al. Identification of homocysteine-suppressive mitochondrial ETC complex genes and tissue expression profile - novel hypothesis establishment. Redox Biol. 2018;17:70-88. doi:10.1016/j.redox.2018.03.015

29. Kibel A, Selthofer-Relatic K, Drenjancevic I, et al. Coronary microvascular dysfunction in diabetes mellitus. J Int Med Res. 2017;45 (6):1901-1929. doi:10.1177/0300060516675504

30. Yang J, Park Y, Zhang H, et al. Feed-forward signaling of TNF- $\alpha$ and $\mathrm{NF}-\kappa \mathrm{B}$ via IKK- $\beta$ pathway contributes to insulin resistance and coronary arteriolar dysfunction in type 2 diabetic mice. Am J Physiol Heart Circ Physiol. 2009;296(6):1850-1858. doi:10.1152/ ajpheart.01199.2008

31. Verma N, Liu M, Ly H, et al. Diabetic microcirculatory disturbances and pathologic erythropoiesis are provoked by deposition of amyloid-forming amylin in red blood cells and capillaries. Kidney Int. 2020;97(1):143-155. doi:10.1016/j.kint.2019.07.028

32. Di Carli MF, Janisse J, Grunberger G, et al. Role of chronic hyperglycemia in the pathogenesis of coronary microvascular dysfunction in diabetes. J Am Coll Cardiol. 2003;41(8):1387-1393. doi:10.1016/ S0735-1097(03)00166-9

33. Woodman RJ, Watts GF, Playford DA, et al. Oxidized LDL and small LDL particle size are independently predictive of a selective defect in microcirculatory endothelial function in type 2 diabetes. Diabetes Obes Metab. 2005;7(5):612-617. doi:10.1111/j.1463-1326.200 5.00478.x

34. von Scholten BJ, Hasbak P, Christensen TE, et al. Cardiac (82) Rb $\mathrm{PET} / \mathrm{CT}$ for fast and non-invasive assessment of microvascular function and structure in asymptomatic patients with type 2 diabetes. Diabetologia. 2016;59(2):371-378. doi:10.1007/s00125015-3799-x

35. Nitenberg A, Valensi P, Sachs R, et al. Impairment of coronary vascular reserve and $\mathrm{ACh}$-induced coronary vasodilation in diabetic patients with angiographically normal coronary arteries and normal left ventricular systolic function. Diabetes. 1993;42(7):1017-1025. doi: $10.2337 /$ diab.42.7.1017

36. Yokoyama I, Momomura S, Ohtake T, et al. Reduced myocardial flow reserve in non-insulin-dependent diabetes mellitus. J Am Coll Cardiol. 1997;30(6):1472-1477. doi:10.1016/S0735-1097(97)00327-6

37. He W, Huang Y, Zhang Y, et al. Cardiac rehabilitation therapy for coronary slow flow phenomenon. Herz. 2020;45(5):468-474. doi:10.1007/s00059-018-4742-y

38. Löffler AI, Bourque JM. Coronary microvascular dysfunction, microvascular angina, and management. Curr Cardiol Rep. 2016;18(1):1. doi:10.1007/s11886-015-0682-9

39. Mittal SR. Diagnosis of coronary microvascular dysfunction - present status. Indian Heart J. 2015;67:552-560.

40. Evrengul H, Tanriverdi H, Kuru O, et al. Elevated homocysteine levels in patients with slow coronary flow: relationship with Helicobacter pylori infection. Helicobacter. 2007;12(4):298-305. doi:10.1111/j.1523-5378.2007.00505.x 


\section{Publish your work in this journal}

The International Journal of General Medicine is an international, peer-reviewed open-access journal that focuses on general and internal medicine, pathogenesis, epidemiology, diagnosis, monitoring and treatment protocols. The journal is characterized by the rapid reporting of reviews, original research and clinical studies across all disease areas. The manuscript management system is completely online and includes a very quick and fair peer-review system, which is all easy to use. Visit http://www.dovepress.com/ testimonials.php to read real quotes from published authors. 